\title{
TRATAMENTO DE ÁGUA UTILIZANDO COAGULANTE MAGNÉTICO BASEADO EM EXTRATO DE SEMENTES DE Moringa oleifera Lam E NANOPARTÍCULAS DE ÓXIDO DE FERRO
}

\author{
T.R.T. SANTOS ${ }^{1 *}$, L. NISHI ${ }^{1}$, D. MANTOVANI ${ }^{1}$, A.M.S. VIEIRA ${ }^{2}$, R. \\ BERGAMASCO $^{1}$, M. F. VIEIRA ${ }^{1}$ \\ ${ }^{1}$ Universidade Estadual de Maringá, Departamento de Engenharia Química \\ ${ }^{2}$ Universidade Estadual de Maringá, Departamento de Engenharia de Alimentos \\ *e-mail: tassia_tonial@hotmail.com
}

\begin{abstract}
RESUMO
Nas estações de tratamento de água, os coagulantes mais empregados são os inorgânicos, que são sais trivalentes de ferro e alumínio. Entretanto, a utilização de substâncias químicas para tornar a água potável pode acarretar outras contaminações, como os subprodutos gerados no processo de desinfecção por cloração, os trihalometanos, substâncias com potencial carcinogênico. Além disto, o alumínio não é biodegradável, podendo ocasionar problemas de disposição e tratamento do lodo gerado. Neste contexto, propõe-se avaliar a eficácia da coagulação/floculação (C/F) utilizando o coagulante natural Moringa oleifera Lam (Mo), funcionalizada com nanopartículas magnéticas de oxido de ferro, para obtenção de flocos que sejam atraídos por um campo magnético externo, permitindo assim uma ágil decantação e separação do líquido clarificado. Os ensaios foram realizados em Jar Test, utilizando água proveniente da bacia do rio Pirapó, Maringá, PR. Amostras foram coletadas para avaliar a eficiência de remoção dos parâmetros de cor aparente, turbidez e compostos com absorção de $\mathrm{UV}_{254 \mathrm{~nm}}$. Foi possível constatar que o tratamento de $\mathrm{C} / \mathrm{F}$ utilizando o coagulante Mo funcionalizada foi capaz de reduzir os valores dos parâmetros físicoquímicos avaliados sob influencia de um campo magnético, com reduzido tempo de sedimentação.
\end{abstract}

\section{INTRODUÇÃO}

O fornecimento de água potável a partir de fontes de água bruta, na maioria das vezes, envolve a utilização de coagulantes introduzidos durante o processo de $\mathrm{C} / \mathrm{F}$ para remoção de turbidez e demais parâmetros de qualidade. Coagulantes químicos, tais como sais de alumínio $\left(\mathrm{AICI}_{3}\right)$, e cloreto férrico $\left(\mathrm{FeCl}_{3}\right)$ são frequentemente utilizados para aumentar a eficiência do processo de $\mathrm{C} / \mathrm{F}$ (SCIBAN et al. 2009).

No entanto, uma vez que se utilizam produtos químicos no tratamento de água existe a possibilidade da formação de subprodutos de desinfecção os quais podem acarretar danos a saúde humana e, além disso, os coagulantes químicos não são biodegradáveis podendo causar sérios problemas em relação à disposição e o tratamento do lodo gerado (BUDD et al. 2004).

Considerando a importância da água potável no mundo, e tendo em mente as preocupações sobre a viabilidade de práticas recentes para atender as crescentes demandas de água, há uma necessidade urgente de desenvolver novas tecnologias e materiais que 
associados com coagulantes naturais possam substituir ou reduzir a utilização de produtos químicos no tratamento de água (CLOETE et al. 2010).

O surgimento da nanotecnologia foi identificado como uma proposta promissora que pode desempenhar um papel importante no fornecimento de água potável. O uso de coagulantes naturais, como o coagulante Moringa oleifera Lam (Mo) associada a nanomateriais é uma maneira inovadora de se melhorar o desempenho da atividade coagulante podendo assim reduzir o seu tempo de sedimentação entre outros fatores (MIKHAYLOVA et al. 2004; LI et al. 2008).

Essa combinação pode oferecer diversas vantagens, tais como, maior eficiência e rapidez na etapa de sedimentação, redução significativa do volume de lodo gerado e possibilidade de reutilização do material coagulante, constituindo dessa forma uma alternativa excelente para ser utilizada nos processos de tratamento de água.

Assim, no presente trabalho, o objetivo foi avaliar a eficácia da coagulação/floculação utilizando o coagulante natural Mo, funcionalizada com nanopartículas magnéticas de oxido de ferro, para obtenção de flocos que sejam atraídos por um campo magnético externo, permitindo assim uma ágil decantação e separação do líquido clarificado.

\section{MATERIAIS E MÉTODOS}

Para os ensaios de $\mathrm{C} / \mathrm{F}$, foram utilizadas amostras de água superficial bruta (AB) provenientes do rio Pirapó, captada pela Companhia de Saneamento do Paraná (SANEPAR) de Maringá-PR, cujas características estão presentes na Tabela 1 . Essa água foi submetida ao processo de $\mathrm{C} / \mathrm{F}$, utilizando como coagulante solução padrão salina do coagulante natural de sementes de Mo, combinadas com nanopartículas de óxido de ferro.
Tabela 1 - Caracterização da Água Bruta

\begin{tabular}{|c|c|}
\hline Parâmetros & Valores \\
\hline Cor aparente $(\mathrm{uH})^{(1)}$ & 265 \\
\hline Turbidez (NTU) & 79 \\
\hline $\mathrm{UV}_{254 \mathrm{~nm}}$ & 0,155 \\
\hline unidade Hazen $=(\mathrm{m}$ & \\
\hline
\end{tabular}

\subsection{Síntese das Nanopartículas Magnéticas}

A síntese das nanopartículas de óxido de ferro foi realizada pelo método sol-gel modificado (SILVA et al. 2013). Neste método, a solução de poliálcool vinílico (PVA) é agitada em temperatura ambiente e após adiciona-se a solução saturada de nitrato de ferro, numa razão molar íon Fe: unidade monomérica do PVA de 1:18. Mantém-se a mistura sob agitação e, então, eleva-se a temperatura para evaporação da água e queima do polímero, obtendo-se o pó precursor. Este material precursor é calcinado em mufla, obtendo-se assim o óxido de ferro nanoparticulado.

\subsection{Preparo da Solução Coagulante Magnética}

As sementes maduras de Mo utilizadas, foram provenientes da Universidade Federal de Sergipe (UFS) removidas da vagem seca, descascadas manualmente e trituradas em liquidificador domestico.

A partir da amostra seca, $0,5 \mathrm{~g}, 1,0 \mathrm{~g}$ e $2 \mathrm{~g}$ do pó de sementes de Mo foram misturado à $100 \mathrm{~mL}$ da solução salina $(\mathrm{NaCl}-1 \mathrm{M})$ utilizando um agitador magnético e filtrados à vácuo em filtro qualitativo obtendo uma solução $1 \%$ (considerando a massa inicial utilizada) da fração solúvel em solução salina de sementes de Mo (MADRONA et al. 2010).

Da solução de Mo foram adicionadas 20 $\mathrm{mL}$ a $1 \mathrm{~mL}$ de óxido de ferro cuja dispersão foi preparada diluindo diferentes concentrações de $\mathrm{Fe}_{2} \mathrm{O}_{3}(0,125 \mathrm{mg}, 0,25 \mathrm{mg}$ e $0,50 \mathrm{mg}$ ) em $2,5 \mathrm{~mL}$ de água destilada e agitada, até completa homogeneização. A 
mistura (óxido de ferro + solução Mo) denominada de $\mathrm{MoFe}$ foi então agitada em temperatura ambiente para posterior utilização. As diferentes concentrações de Mo e $\mathrm{Fe}$ foram combinadas e uma letra foi determinada para cada diferente combinação como representadas na Tabela 2.

Tabela 2 - Combinação de quantidades de $\mathrm{Fe}$ e Mo e suas letras representativas

\begin{tabular}{ccc}
\hline & $\mathrm{Fe}_{2} \mathrm{O}_{3}(\mathrm{mg})$ & $\mathrm{Mo}(\mathrm{g})$ \\
\hline $\mathrm{B}$ & 0 & 0 \\
$\mathrm{C}$ & 0 & 0,5 \\
$\mathrm{D}$ & 0 & 2 \\
$\mathrm{E}$ & 0 & 0 \\
$\mathrm{~F}$ & 0,125 & 0,5 \\
$\mathrm{G}$ & 0,125 & 1 \\
$\mathrm{H}$ & 0,125 & 2 \\
$\mathrm{I}$ & 0,125 & 0 \\
$\mathrm{~J}$ & 0,25 & 0,5 \\
$\mathrm{~K}$ & 0,25 & 1 \\
$\mathrm{~L}$ & 0,25 & 2 \\
$\mathrm{M}$ & 0,25 & 0 \\
$\mathrm{~N}$ & 0,5 & 0,5 \\
$\mathrm{O}$ & 0,5 & 1 \\
$\mathrm{P}$ & 0,5 & 2 \\
\hline $\mathrm{F}$ & 0,5 &
\end{tabular}

Fonte: Autor (2015).

\subsection{Ensaios em Jar Test}

Os ensaios de $\mathrm{C} / \mathrm{F}$ foram realizados em equipamento de Teste de Jarros (Jar Test) da Nova Ética - Modelo 218 LDB. A agitação foi proporcionada por agitadores situados na parte superior do equipamento, e controlada por um seletor de rotações (0 a $150 \mathrm{rpm}$ ).

As condições de operação (tempo de coagulação, gradiente de velocidade rápida, tempo de floculação e gradiente de velocidade lenta) foram otimizadas por MADRONA et al. (2010) (Tabela 3). A sedimentação foi avaliada sob a influência de um campo magnético externo, de forma a acelerar este processo.

Tabela 3 - Condições de Operação no Jar Test Condições de operação

Gradiente de mistura rápida $\left(\mathrm{s}^{-1}\right) \quad 690$

Tempo de mistura rápida ( $\mathrm{min}) \quad 3,0$

Gradiente de mistura lenta $\left(\mathrm{s}^{-1}\right) \quad 23$

Tempo de mistura lenta (min) 15

Tempo de sedimentação (min) $\quad 30$

(MADRONA et al. 2010)

Fonte: Autor (2015).

\subsection{Análises Físico-Químicas}

\subsubsection{Turbidez}

A medida é feita pelo princípio nefelométrico, que consiste na leitura de intensidade de luz desviada pelas partículas num angulo de $90^{\circ}$ em relação à luz incidente.

A medida da turbidez foi feita em turbidímetro PoliControl, modelo AP2000 e utilizando soluções padrão de formazina. Os resultados foram expressos em unidades nefelométricas de turbidez (NTU).

\subsubsection{Cor Aparente}

A cor foi medida em espectrofotômetro Hach modelo DR/2000, programa 120, comprimento de onda de $455 \mathrm{~nm}$, por comparação visual com padrão de cobaltoplatina, segundo procedimento recomendado pelo Standard Methods (APHA 2005).

\subsubsection{Compostos com Absorção em $U V_{254 n m}$ (UV 254nm)}

A análise dos compostos com absorção em $U_{254 n m}$ foi determinada em espectrofotômetro BEL, modelo 2000UV, segundo procedimento recomendado pelo Standard Methods (APHA 2005), onde consta que a seleção do comprimento de onda no ultravioleta é arbitrária, historicamente 
utiliza-se 254nm, desde que a mesma minimize a interferência de outros compostos.

\section{RESULTADOS E DISCUSSÕES}

Foram analisados os parâmetros cor aparente (Figura 1), turbidez (Figura 2) e compostos com absorção em $\mathrm{UV}_{254 \mathrm{~nm}}$ (Figura 3) utilizando o coagulante MoFe, para avaliar a sua capacidade de remoção desses parâmetros de qualidade.

Figura 1 - Avaliação do parâmetro cor 1parente, utilizando o coagulante $\mathrm{MoFe}$ sem e sob a influência de campo magnético

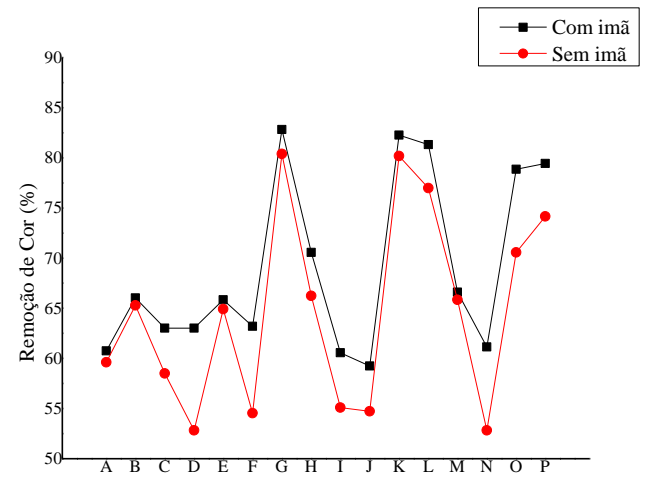

Em relação a cor aparente (Figura 1), foram obtidas remoções em torno de $85 \%$ utilizando a combinação $\mathrm{G}$ de funcionalização $(0,125 \mathrm{mg}$ de Fe e $1 \mathrm{~g}$ de Mo) sob influencia de campo magnético.

Figura 2 - Avaliação do parâmetro turbidez, utilizando o coagulante $\mathrm{MoFe}$ sem e sob a influência de campo magnético

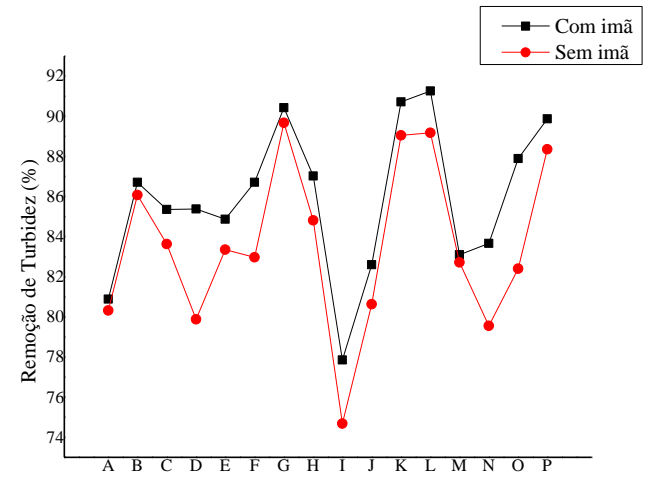

Quanto ao parâmetro turbidez (Figura 2), a remoção alcançada foi em torno de $90 \%$. De forma semelhante ao parâmetro cor aparente, a melhor combinação de funcionalização do coagulante foi a $\mathrm{G}(0,125$ $\mathrm{mg}$ de $\mathrm{Fe}$ e $1 \mathrm{~g}$ de Mo), também sob influência do campo magnético.

Figura 3 - Avaliação do parâmetro $U_{254 \mathrm{~nm}}$ utilizando o coagulante MoFe sem e sob a influencia de campo magnético

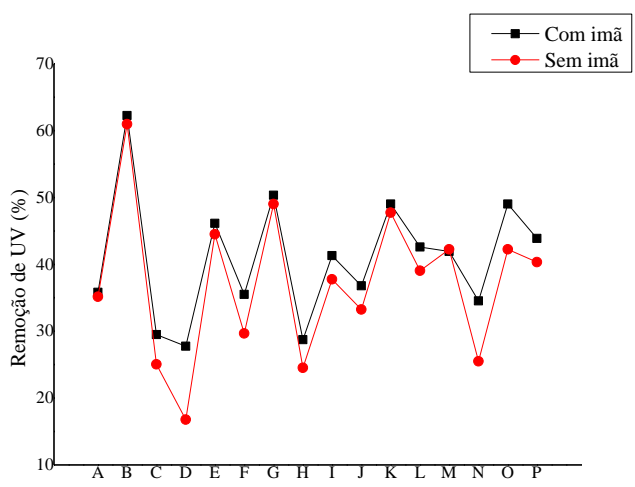

Para o parâmetro $U_{254 n m}$ (Figura 3), a maior porcentagem de remoção alcançada foi em torno de $50 \%$, utilizando a combinação $\mathrm{G}$ $(0,125 \mathrm{mg}$ de Fe e $1 \mathrm{~g}$ de Mo) sob influência do campo magnético.

Diante de todos os parâmetros avaliados podemos observar que o estudo utilizando $A B$ tratada com $\mathrm{MoFe}$, revelou uma redução significativa dos parâmetros avaliados, em um curto intervalo de tempo (30 min), utilizando um campo magnético externo, em comparação com 60 minutos necessários para sedimentação por gravidade normalmente utilizados quando utiliza-se o coagulante natural Mo (CARDOSO et al. 2008; MADRONA et al. 2010). Isto pode ser explicado pelas teorias da curva de magnetização, que coloca que quando as nanopartículas estão dentro de um campo magnético externo constante, o momento magnético interno girará na mesma direção do campo magnético externo. Esta ocorrência vai aumentar as propriedades magnéticas das 
nanopartículas causando a agregação das impurezas da água melhorando assim o processo de C/F (LU, SALABAS,SCHÜTH 2007; FARAJI, YAMINI,REZAEE 2010)

\section{CONCLUSÕES}

$\mathrm{O}$ tratamento de $\mathrm{C} / \mathrm{F}$ utilizando o coagulante $\mathrm{MoFe}$ com a combinação $\mathrm{G}(0,125$ $\mathrm{mg}$ de $\mathrm{Fe}$ e $1 \mathrm{~g}$ de Mo) foi capaz de reduzir os valores dos parâmetros físico-químicos avaliados sob influência de um campo magnético externo, reduzindo tempo de sedimentação.

\section{NOMENCLATURA}

Coagulação/floculação - C/F.

Moringa oleifera Lam - Mo.

Água superficial bruta - AB.

Óxido de ferro + solução de Mo MoFe.

\section{REFERÊNCIAS}

APHA, Standard methods for the examination of water and wastewater, in American Public Health Association (APHA): Washington, DC, USA, 2005.

BUDD, G. C., HESS, A. F., et al. Coagulation applications for new treatment goals. Journal (American Water Works Association), v. 96, p. 102-113, 2004.

CARDOSO, K. C., BERGAMASCO, R., et al. Otimizing mixture and decantation times in the process of coagulation/flocculation of raw water using Moringa oleifera Lam. Acta Scientiarum-Technology, v. 30, p. 193-198, 2008.

CLOETE, T. E., KWAADSTENIET, M., et al. Nanotechnology in Water Treatment Applications. Norfolk, Caister Academic Press 2010.
FARAJI, M., YAMINI, Y., et al. Magnetic nanoparticles: Synthesis, stabilization, functionalization, characterization, and applications. Journal of the Iranian Chemical Society, v. 7, p. 1-37, 2010.

LI, Q., MAHENDRA, S., et al. Antimicrobial nanomaterials for water disinfection and microbial control: Potential applications and implications. Water Research, v. 42, p. 4591-4602, 2008.

LU, A.-H., SALABAS, E. L., et al. Magnetic Nanoparticles: Synthesis, Protection, Functionalization, and Application. Angewandte Chemie International Edition, v. 46, p. 1222-1244, 2007.

MADRONA, G. S., SERPELLONI, G. B., et al. Study of the Effect of Saline Solution on the Extraction of the Moringa oleifera Seed's Active Component for Water Treatment. Water Air and Soil Pollution, v. 211, p. 409-415, 2010.

MIKHAYLOVA, M., KIM, D. K., et al. Superparamagnetism of Magnetite Nanoparticles: Dependence on Surface Modification. Langmuir, v. 20, p. 24722477, 2004.

SCIBAN, M., KLASNJA, M., et al. Removal of water turbidity by natural coagulants obtained from chestnut and acorn. Bioresource Technology, v. 100, p. 66396643, 2009.

SILVA, M. F., DE OLIVEIRA, L. A. S., et al. Nanometric particle size and phase controlled synthesis and characterization of $\mathrm{g}-\mathrm{Fe}_{2} \mathrm{O}_{3}$ or $(\mathrm{a}+\mathrm{g})-\mathrm{Fe}_{2} \mathrm{O}_{3}$ by a modified sol-gel method. Journal of Applied Physics, v. 114, p. 104311-104317, 2013.

\section{AGRADECIMENTOS}


Os autores agradecem à Capes e Fundação Araucária pelo apoio financeiro; à Universidade Federal de Sergipe (UFS), pela doação das sementes de Mo; e a SANEPAR, pelas amostras de $\mathrm{AB}$ fornecidas. 